\title{
Copulas: an open field for risk management
}

March 23, 2001

\section{Introduction}

One of the main issues of risk management is the aggregation of individual risks. A powerful concept to aggregate the risks - the copula function - has been introduced in finance by EMBRECHTS, MCNEIL, and STRAumAnN [1999,2000]. In their papers, the authors clarify the essential concepts of dependence and correlation and certainly will greatly influence the risk management industry. The goal of this paper is to provide simple applications for the practical use of copulas for risk management from an industrial point of view. First, we remind some basics about copulas. Then, some applications of copulas for market risk, credit risk and operational risk are given. We will not provide a full mathematical treatment of the subject and we refer interested readers to JoE [1997] or NELSEN [1999].

\section{Copulas definition}

A copula is a function that links univariate margins to the full multivariate distribution. Then, this function is the joint distribution function of $N$ standard uniform random variables. Mathematically speaking, a function $\mathbf{C}$ is a copula function if it fulfills the following properties (NELSEN [1999]):

1. $\operatorname{Dom} \mathbf{C}=[0,1]^{N}$;

2. C is grounded and $N$-increasing ${ }^{1}$;

3. The margins $\mathbf{C}_{n}$ of $\mathbf{C}$ satisfy $\mathbf{C}_{n}(u)=$ $\mathbf{C}(1, \ldots, 1, u, 1, \ldots, 1)=u$ for all $u$ in $[0,1]$.

This class of function is very important because it permits to define the dependence structure between the margins of a multivariate distribution. Indeed, let think about $N$ random variables $\left(X_{1}, \ldots, X_{N}\right)$ with

\footnotetext{
${ }^{1}$ These properties mean that $\mathbf{C}$ is a positive probability measure.
}

multivariate distribution $\mathbf{F}$ and univariate margins $\left(\mathbf{F}_{1}, \ldots, \mathbf{F}_{N}\right)$. Then we have the canonical decomposition

$$
\mathbf{F}\left(x_{1}, \ldots, x_{N}\right)=\mathbf{C}\left(\mathbf{F}_{1}\left(x_{1}\right), \ldots, \mathbf{F}_{N}\left(x_{N}\right)\right)
$$

Moreover, Abe Sklar proved in 1959 that the copula $\mathbf{C}$ is unique for a given distribution $\mathbf{F}$ if the margins are continuous. To illustrate the idea behind the copula function, we can think about the multivariate gaussian that is a 'standard' assumption in risk management. To postulate that a vector $\left(X_{1}, \ldots, X_{N}\right)$ is gaussian is equivalent to assume that:

1. the univariate margins $\mathbf{F}_{1}, \ldots, \mathbf{F}_{N}$ are gaussians;

2. these margins are linked by a unique copula function $\mathbf{C}$ (called Normal copula) such that:

$$
\mathbf{C}_{\boldsymbol{\rho}}\left(u_{1}, \ldots, u_{N}\right)=\boldsymbol{\Phi}_{\boldsymbol{\rho}}\left(\Phi^{-1}\left(u_{1}\right), \ldots, \Phi^{-1}\left(u_{N}\right)\right)
$$

with $\boldsymbol{\Phi}_{\rho}$ the multivariate normal cdf with correlation matrix $\rho$ and $\Phi^{-1}$ the inverse of the standard univariate gaussian distribution.

It appears that the risk can be splitted into two parts: the individual risks and the dependence structure between them. Indeed, the assumption of normality for the margins can be removed and $\mathbf{F}_{1}, \ldots, \mathbf{F}_{N}$ may be fat-tailed distributions (e.g. Student, Weibull, Pareto) and the dependence may still be characterized by a Normal copula. This leads to a new multivariate distribution that takes into account, for example, the leptokurtic property of asset returns. This is illustrated by Figure 1.

From standard textbooks, we know that the density $f$ of the distribution $\mathbf{F}$ is its $N$-derivative, if it exists:

$$
f\left(x_{1}, \ldots, x_{N}\right)=\frac{\partial \mathbf{F}\left(x_{1}, \ldots, x_{N}\right)}{\partial x_{1} \cdots \partial x_{N}}
$$




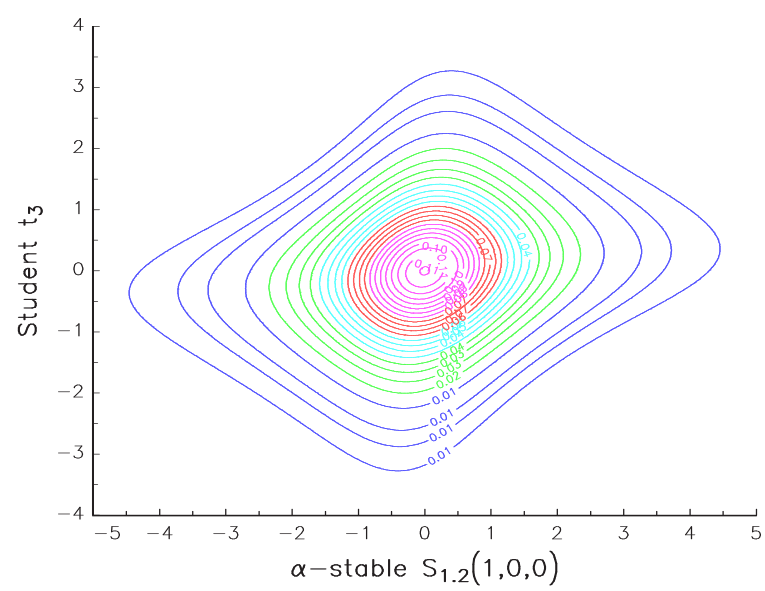

Figure 1: Contour of the bivariate density of two random variables with the first margin $\alpha$-stable, the second margin Student and a Normal copula with $\rho=0.3$.

Let $f_{n}$ be the density function that corresponds to the $n$-th margin. The expression of the copula density $c$ is

$$
c\left(u_{1}, \ldots, u_{N}\right)=\frac{\partial \mathbf{C}\left(u_{1}, \ldots, u_{N}\right)}{\partial u_{1} \cdots \partial u_{N}}
$$

It comes that the canonical decomposition of the density of $\mathbf{F}$ is

$f\left(x_{1}, \ldots, x_{N}\right)=c\left(\mathbf{F}_{1}\left(x_{1}\right), \ldots, \mathbf{F}_{N}\left(x_{N}\right)\right) \times \prod_{n=1}^{N} f_{n}\left(x_{n}\right)$

The formula of the Normal copula density is obtained by derivating equation (1):

$$
c\left(u_{1}, \ldots, u_{N} ; \boldsymbol{\rho}\right)=\frac{1}{|\boldsymbol{\rho}|^{\frac{1}{2}}} \exp \left(-\frac{1}{2} \boldsymbol{\varsigma}^{\top}\left(\boldsymbol{\rho}^{-1}-\mathbb{I}\right) \boldsymbol{\varsigma}\right)
$$

with $\boldsymbol{\varsigma}=\left(\boldsymbol{\varsigma}_{1}, \ldots, \boldsymbol{\varsigma}_{N}\right)$ where $\boldsymbol{\varsigma}_{n}=\boldsymbol{\Phi}^{-1}\left(u_{n}\right)$ for $n=$ $1, \ldots, N$. Other type of dependence could be postulated, for example the Student copula which has the following density ${ }^{2}$

$$
|\boldsymbol{\rho}|^{-\frac{1}{2}} \frac{\Gamma\left(\frac{\nu+N}{2}\right)\left[\Gamma\left(\frac{\nu}{2}\right)\right]^{N}}{\left[\Gamma\left(\frac{\nu+1}{2}\right)\right]^{N} \Gamma\left(\frac{\nu}{2}\right)} \frac{\left(1+\frac{1}{\nu} \boldsymbol{\varsigma}^{\top} \boldsymbol{\rho}^{-1} \boldsymbol{\varsigma}\right)^{-\frac{\nu+N}{2}}}{\prod_{n=1}^{N}\left(1+\frac{\varsigma_{n}^{2}}{\nu}\right)^{-\frac{\nu+1}{2}}}
$$

with $\varsigma_{n}=t_{\nu}^{-1}\left(u_{n}\right), \Gamma$ the gamma function and $\nu$ the degrees of freedom.

To illustrate the difference between the Normal copula and the Student copula, we have plotted bivariate

\footnotetext{
${ }^{2}$ We refer to Bouyé, Durrleman, Nickeghbali, Riboulet and RonCALli [2000] for the proof.
}

simulations (i) with gaussian margins (ii) with normalized Student margins (such that the variances are the same).
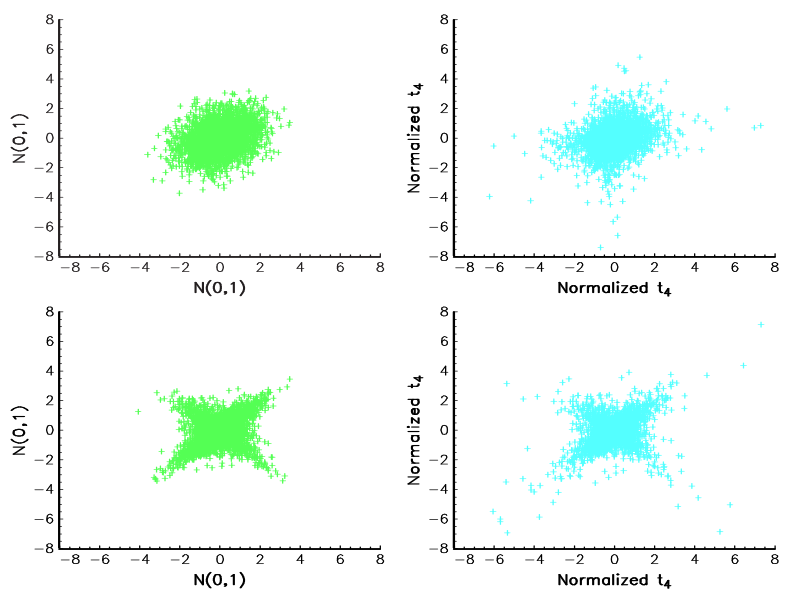

Figure 2: Monte Carlo simulation (5000 simulations) with respectively a Normal copula $(\rho=0.3)$ - upper plots - and a Student copula $(\rho=0.3, \nu=1)$ - lower plots. The left plots assume gaussian margins whereas the right plots assume normalized Student margins.

\section{Market risk management}

The copula methodology can be applied both to compute Value at Risk ( $V a R$ ) and to perform stress testing. The two approaches are explained.

\section{1 $\quad V a R$ for portfolios}

As noted by Embrechts, MCNeIL and Straumann [2000], the correlation is a special case through all measures that are available to understand the relationships between all the risks. If we assume a Normal copula, the empirical correlation is a good measure of the dependence only if the margins are gaussians. To illustrate this point, we can construct two estimators:

1. The empirical correlation $\hat{\boldsymbol{\rho}}$;

2. The canonical correlation $\hat{\boldsymbol{\rho}}_{\mathrm{CML}}$ obtained as follows: the data are mapped to empirical uniforms and transformed with the inverse function of the gaussian distribution. The correlation is then computed for the transformed data ${ }^{3}$.

${ }^{3} \hat{\boldsymbol{\rho}}_{\mathrm{CML}}$ is also called the 'omnibus estimator'. It is consistent and asymptotically normally distributed (GENEST, GHOUDI and RIVEST [1995]). 
The advantage of the canonical measure is that no distribution is assumed for individual risks. Indeed, it can be shown that a misspecification about the marginal distributions (for example to assume gaussian margins if they are not) leads to a biased estimator of the correlation matrix. This is illustrated by the following example for asset returns. The database of the London Metal Exchange ${ }^{4}$ is used and the spot prices of the commodities Aluminium Alloy (AL), Copper (CU), Nickel (NI), Lead (PB) and the 15 months forward prices of Aluminium Alloy (AL-15), dating back to January 1988, are considered. The two correlation measures of asset returns are reported below ${ }^{5}$.

\begin{tabular}{c|ccccc} 
& $\mathrm{AL}$ & $\mathrm{AL}-15$ & $\mathrm{CU}$ & $\mathrm{NI}$ & $\mathrm{PB}$ \\
\hline $\mathrm{AL}$ & 1.00 & 0.82 & 0.44 & 0.36 & 0.33 \\
$\mathrm{AL}-15$ & & 1.00 & 0.39 & 0.34 & 0.30 \\
$\mathrm{CU}$ & & & 1.00 & 0.37 & 0.31 \\
$\mathrm{NI}$ & & & & 1.00 & 0.31 \\
$\mathrm{~PB}$ & & & & & 1.00
\end{tabular}

Table 1: Correlation matrix $\hat{\boldsymbol{\rho}}$ of the LME data

\begin{tabular}{l|lllll} 
& $\mathrm{AL}$ & $\mathrm{AL}-15$ & $\mathrm{CU}$ & $\mathrm{NI}$ & $\mathrm{PB}$ \\
\hline $\mathrm{AL}$ & 1.00 & 0.85 & 0.49 & 0.39 & 0.35 \\
$\mathrm{AL}-15$ & & 1.00 & 0.43 & 0.35 & 0.32 \\
$\mathrm{CU}$ & & & 1.00 & 0.41 & 0.36 \\
$\mathrm{NI}$ & & & & 1.00 & 0.33 \\
$\mathrm{~PB}$ & & & & & 1.00
\end{tabular}

Table 2: Correlation matrix $\hat{\boldsymbol{\rho}}_{\mathrm{CML}}$ of the LME data

\begin{tabular}{l|lllll}
$\nu=2$ & $\mathrm{AL}$ & $\mathrm{AL}-15$ & $\mathrm{CU}$ & $\mathrm{NI}$ & $\mathrm{PB}$ \\
\hline $\mathrm{AL}$ & 1.00 & 0.82 & 0.33 & 0.25 & 0.19 \\
$\mathrm{AL}-15$ & & 1.00 & 0.27 & 0.22 & 0.16 \\
$\mathrm{CU}$ & & & 1.00 & 0.27 & 0.22 \\
$\mathrm{NI}$ & & & & 1.00 & 0.20 \\
$\mathrm{~PB}$ & & & & & 1.00
\end{tabular}

Table 3: Correlation matrix $\hat{\boldsymbol{\rho}}_{\mathrm{ML}}$ with Student copula $(\nu=1)$ of the LME data

Even if we assume that the margins are gaussians, we will show that the choice of the dependence structure has a great impact on the $V a R$ computation of a portfolio. If we consider that the dependence of the LME

\footnotetext{
${ }^{4}$ The database is available on the web site of the LME http://www.Ime.co.uk.

${ }^{5}$ The standard errors are not reported here. However, the correlations of Table 2 and Table 3 are in italics if they are significantly different from Table 1 at $5 \%$ confidence level.
}

data is a Student copula with 1 degree of freedom ${ }^{6}$, the obtained parameter matrix (see Table 3) differs from the Normal one ${ }^{7}$ of Table 1. Then, let consider a portfolio a with $\mathbf{P}(t)$ the price vector of the assets at time $t$. The one period value-at-risk with $\alpha$ confidence level is defined by $V a R=\mathbf{F}^{-1}(1-\alpha)$ with $\mathbf{F}$ the distribution of the random variate $\mathbf{a}^{\top}(\mathbf{P}(t+1)-\mathbf{P}(t))$. Let assume we have three different portfolios (a negative number corresponds to a short position):

\begin{tabular}{c|ccccc} 
& $\mathrm{AL}$ & $\mathrm{AL}-15$ & $\mathrm{CU}$ & $\mathrm{NI}$ & $\mathrm{PB}$ \\
\hline $\mathrm{P}_{1}$ & 1 & 1 & 1 & 1 & 1 \\
$\mathrm{P}_{2}$ & -1 & -1 & -1 & 1 & 1 \\
$\mathrm{P}_{3}$ & 2 & 1 & -3 & 4 & 5
\end{tabular}

For these three portfolios, we assume that the margins are gaussians and compare ${ }^{8}$ the VaRs under the assumption of Normal copula and Student copula with $\nu=1$. The higher the quantile ${ }^{9}$, the more the Student dependence leads to a higher $V a R$ (see Tables 4 and 5). An interesting point is that for the three portfolios and for a low level quantile (for example 90\%), the Student copula leads to lower VaRs. In Table 6, we have reported the $\mathrm{VaR}$ when the copula is Normal and the margins are Student. If we compare this table with Table 4, we remark the impact of the choice of fattailed distributions on the $V a R$ computation ${ }^{10}$. Note that if no analytical formula is available for the $V a R$ computation, the results are obtained by simulation.

\begin{tabular}{c|ccccc} 
& $90 \%$ & $95 \%$ & $99 \%$ & $99.5 \%$ & $99.9 \%$ \\
\hline $\mathrm{P}_{1}$ & 7.26 & 9.33 & 13.14 & 14.55 & 17.45 \\
$\mathrm{P}_{2}$ & 4.04 & 5.17 & 7.32 & 8.09 & 9.81 \\
$\mathrm{P}_{3}$ & 13.90 & 17.82 & 25.14 & 27.83 & 33.43
\end{tabular}

Table 4: VaR with Normal copula

\begin{tabular}{c|ccccc} 
& $90 \%$ & $95 \%$ & $99 \%$ & $99.5 \%$ & $99.9 \%$ \\
\hline $\mathrm{P}_{1}$ & 5.69 & 7.95 & 13.19 & 15.38 & 20.06 \\
$\mathrm{P}_{2}$ & 3.82 & 5.55 & 9.75 & 11.65 & 16.41 \\
$\mathrm{P}_{3}$ & 13.41 & 19.36 & 34.16 & 40.55 & 54.48
\end{tabular}

Table 5: VaR with Student copula $(\nu=1)$

\footnotetext{
${ }^{6}$ We use the iterative algorithm described in [2] and [5] to estimate the parameters matrix $\rho$.

${ }^{7}$ But if $\nu$ is equal 2 , only three parameters among eleven are significantly different at $5 \%$ confidence level.

${ }^{8}$ All the parameters are estimated using maximum likelihood method.

${ }^{9}$ We have reported the $V a R$ for the $99.9 \%$ quantile, which approximately corresponds to the rating target $\mathrm{A}$.

${ }^{10}$ For low level quantiles $(90 \%$ and $95 \%$ ), we have lower $\operatorname{VaRs}$ whereas higher quantiles produce bigger VaRs.
} 


\begin{tabular}{c|ccccc} 
& $90 \%$ & $95 \%$ & $99 \%$ & $99.5 \%$ & $99.9 \%$ \\
\hline $\mathrm{P}_{1}$ & 6.51 & 8.82 & 14.26 & 16.94 & 24.09 \\
$\mathrm{P}_{2}$ & 3.77 & 5.00 & 7.90 & 9.31 & 13.56 \\
$\mathrm{P}_{3}$ & 12.76 & 17.05 & 27.51 & 32.84 & 49.15
\end{tabular}

Table 6: VaR with Normal copula and Student margins $(\nu=4)$

Finally, we give in Table 7 an idea about the computational time needed to estimate the value-at-risk based on a Normal copula and Student margins. The number of simulations is 100000 and the computation has been done with the GAUSS software and a Pentium III $550 \mathrm{Mhz}$. These times are given for indication since the number of simulations is constant and does not depend on the number of assets (the problem of dimensionality is not treated).

\begin{tabular}{c|c} 
Number of assets & Computational time \\
\hline 2 & $0.1 \mathrm{sc}$ \\
10 & $24.5 \mathrm{sc}$ \\
100 & $4 \mathrm{mn} 7 \mathrm{sc}$ \\
500 & $33 \mathrm{mn} 22 \mathrm{sc}$ \\
1000 & $1 \mathrm{hr} 44 \mathrm{mn} 45 \mathrm{sc}$
\end{tabular}

Table 7: Computational time for computing $V a R$

\section{2 $\quad$ Stress testing}

The extreme value theory is now familiar to practitioners. It allows, for example, to apply stress scenarios to a portfolio. However, the extension to the multivariate case is a difficult issue. There exists a special class of copula function that avoids the problem. Indeed, any copula function $\mathbf{C}_{\star}$ such that

$$
\mathbf{C}_{\star}\left(u_{1}^{t}, \ldots, u_{N}^{t}\right)=\mathbf{C}_{\star}^{t}\left(u_{1}, \ldots, u_{N}\right) \quad \forall t>0
$$

can be used to construct a multivariate extreme value distribution (Deheuvels [1978]). We just write the equations for maxima as the problem is identical for minima. The maxima are defined componentwise

$$
\chi_{m}^{+}=\left(\chi_{1, m}^{+}, \ldots, \chi_{N, m}^{+}\right):=\left(\bigvee_{k=1}^{m} X_{1, k}, \ldots, \bigvee_{k=1}^{m} X_{N, k}\right)
$$

For each maxima $\chi_{n, m}^{+}$, its univariate generalized extreme value (GEV) distribution $\mathbf{G}_{n}$ with

$$
\mathbf{G}_{n}\left(\chi_{n, m}^{+}\right)=\exp \left\{-\left[1+\xi_{n}\left(\frac{\chi_{n, m}^{+}-\mu_{n}}{\sigma_{n}}\right)\right]^{-\frac{1}{\xi_{n}}}\right\}
$$

can be estimated for $n=1, \ldots, N$. Then, the multivariate extreme value distribution for maxima $\mathbf{G}$ is

$$
\mathbf{G}\left(\chi_{1}^{+}, \ldots, \chi_{N}^{+}\right)=\mathbf{C}_{\star}\left(\mathbf{G}_{1}\left(\chi_{1}^{+}\right), \ldots, \mathbf{G}_{N}\left(\chi_{N}^{+}\right)\right)
$$

To illustrate how this result can be used for risk management, we consider an example which focuses on the extremes of the pair (CAC40,DowJones). First, the GEV univariate distributions are estimated for maxima and minima of CAC40 and DowJones respectively (that makes four estimations). Then, let assume a copula that fulfills the condition (2), for example the Gumbel copula:

$$
\mathbf{C}_{\star}\left(u_{1}, u_{2}\right)=\exp \left(-\left(\left(-\ln u_{1}\right)^{\delta}+\left(-\ln u_{2}\right)^{\delta}\right)^{\frac{1}{\delta}}\right)
$$

with $\delta$ the dependence parameter $(\delta=1$ for independence and $\delta=\infty$ for fully dependent extrema). It is then possible to construct a failure area that corresponds to the set of values $\left(\chi_{1}^{+}, \chi_{2}^{+}\right)$such that $\operatorname{Pr}\left\{\chi_{1}^{+}>\chi_{1}, \chi_{2}^{+}>\chi_{2}\right\}=1-\mathbf{G}_{1}\left(\chi_{1}\right)-\mathbf{G}_{2}\left(\chi_{2}\right)+$ $\mathbf{C}_{\star}\left(\mathbf{G}_{1}\left(\chi_{1}\right), \mathbf{G}_{2}\left(\chi_{2}\right)\right)$ equals a given level of probability. By applying the same methodology to the

\begin{tabular}{|c|c|}
\hline & DowJones \\
\hline $\begin{array}{l}\left(\chi_{1}^{-}, \chi_{2}^{+}\right) \\
\lambda=37 \%\end{array}$ & $\begin{array}{l}\left(\chi_{1}^{+}, \chi_{2}^{+}\right) \\
\lambda=42 \%\end{array}$ \\
\hline $\begin{array}{l}\left(\chi_{1}^{-}, \chi_{2}^{-}\right) \\
\lambda=44 \%\end{array}$ & $\begin{array}{l}\left(\chi_{1}^{+}, \chi_{2}^{-}\right) \\
\lambda=33 \%\end{array}$ \\
\hline
\end{tabular}
three other pairs $(\min / \max , \max / \mathrm{min}$ and $\min / \mathrm{min}$ ), one can construct the failure area from the estimation of the dependence for the four quadrants of (CAC40,DowJones)

with $\chi_{1}^{-}$and $\chi_{2}^{-}$the minima. To characterize the dependence of extremal risks, the upper tail dependence coefficient $\lambda$ (see Joe [1997]) is used:

$$
\lambda=\lim _{\alpha \rightarrow 1} \operatorname{Pr}\left\{X_{1}>\mathbf{F}_{1}^{-1}(\alpha) \mid X_{2}>\mathbf{F}_{2}^{-1}(\alpha)\right\}
$$

We can interpret $\lambda$ as the probability that one random variable is extreme given that the other is extreme. In our example, the dependence of minima is not significantly different from the dependence of maxima, which means that bear markets are quite similar to bull markets from an economic point of view. Figure 3 provides an example for a probability that is equivalent to a 5 
years waiting time. We remark that some past extremal events have a waiting time bigger than 5 years.

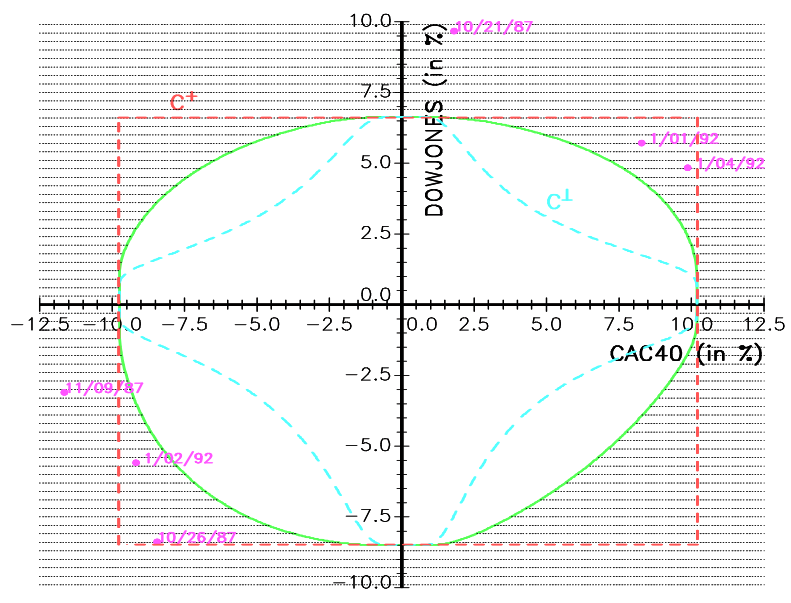

Figure 3: Failure areas for the pair (CAC40,DowJones) and a 5 years waiting time. Note that $\mathbf{C}^{\perp}$ and $\mathbf{C}^{+}$ correspond to the cases of independence and perfect positive dependence between the two asset returns.

\section{Credit risk management}

One of the main issue concerning credit risk is without doubt the modelling of joint default distribution. Li [2000] and MACCARINELLI and Maggiolini [2000] suggest that copulas could be a suitable tool for such a problem. Indeed, a default is generally described by a survival function $\mathbf{S}(t)=\operatorname{Pr}\{T>t\}$, which indicates the probability that a security will attain age $t$. $T$ is a random variable called the survival time, which is denoted time-until-default in Li [2000]. Let $\breve{\mathbf{C}}$ be a survival copula. A multivariate survival distribution $\mathbf{S}$ can be defined as follows

$$
\mathbf{S}\left(t_{1}, \ldots, t_{N}\right)=\breve{\mathbf{C}}\left(\mathbf{S}_{1}\left(t_{1}\right), \ldots, \mathbf{S}_{N}\left(t_{N}\right)\right)
$$

where $\left(\mathbf{S}_{1}, \ldots, \mathbf{S}_{N}\right)$ are the marginal survival functions. NELSEN [1999] notices that "C couples the joint survival function to its univariate margins in a manner completely analogous to the way in which a copula connects the joint distribution function to its margins". Introducing correlation between defaultable securities can then be done using the copula framework.

\begin{tabular}{c|cccccccc}
\cline { 2 - 9 } & \multicolumn{10}{c}{ Final rating } & & & \\
\hline Initial rating & AAA & AA & A & BBB & BB & B & CCC & D \\
\hline AAA & 92.54 & 6.48 & 0.86 & 0.06 & 0.06 & 0.00 & 0.00 & 0.00 \\
AA & 0.63 & 91.87 & 6.64 & 0.65 & 0.06 & 0.11 & 0.04 & 0.00 \\
A & 0.08 & 2.26 & 91.66 & 5.11 & 0.61 & 0.23 & 0.01 & 0.04 \\
BBB & 0.05 & 0.27 & 5.84 & 87.74 & 4.74 & 0.98 & 0.16 & 0.22 \\
BB & 0.04 & 0.11 & 0.64 & 7.85 & 81.14 & 8.27 & 0.89 & 1.06 \\
B & 0.00 & 0.11 & 0.30 & 0.42 & 6.75 & 83.07 & 3.86 & 5.49 \\
CCC & 0.19 & 0.00 & 0.38 & 0.75 & 2.44 & 12.03 & 60.71 & 23.50 \\
\hline
\end{tabular}

Table 8: S\&P one-year transition matrix (in \%)

\subsection{Computing the risk of a credit portfolio}

Using the previous framework, it is then possible to compute risk measure (or economic capital) of any portfolio of risky securities. Thus, one could remark for instance that the CreditMetrics methodology implicitly uses the Normal Copula in (3) for their credit risk measure (LI [2000]). Indeed, in this (structural) approach the distribution of the joint default is obtained from the Asset Value Model of Merton where underlyings are assumed to be gaussian. To show that the dependence function has a great impact on the computation of the risk of a credit portfolio, we consider the example of joint default probability in the CreditMetrics framework with the one-year transition matrix of Table 8. In Figure 4, we remark that even if the copulas has the same Kendall's tau ${ }^{11}$, we can obtain very different joint default probabilities, and of course very different credit risk VaRs.
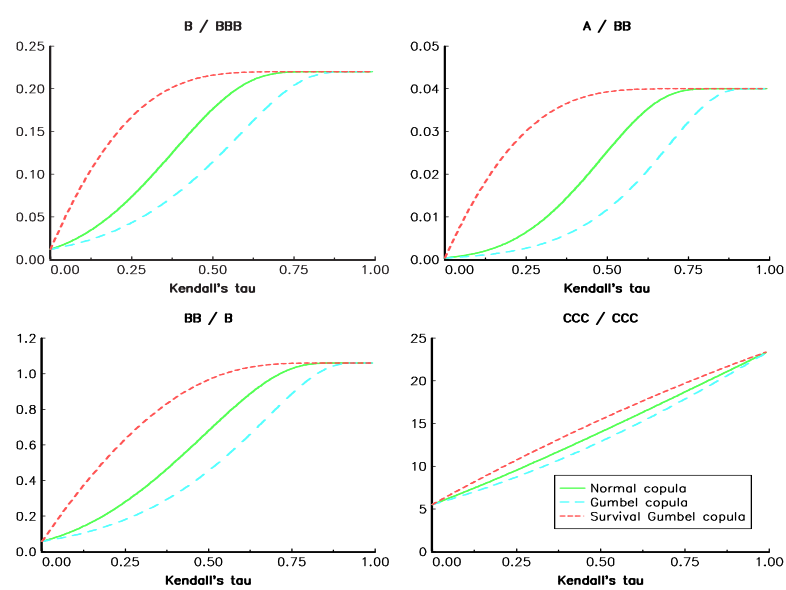

Figure 4: One-year joint default probabilities (in \%). In order to compare them, we use Kendall's tau.

In the case of CreditRisk+, Coutant, Martineu, Messines, Riboulet and Roncalli [2001] show that the dependence function between defaults is related

\footnotetext{
${ }^{11}$ It is one of the most known measure to compare the concordance between copulas.
} 
to a special class of Archimedean copulas called frailty models ${ }^{12}$. For alternative approach, we refer to the works of survival analysis and multivariate exponential distributions which provide a starting point for many extensions (see the survey [9]).

\subsection{Pricing credit derivatives}

Copulas may also apply to the pricing of credit derivatives. One may for instance consider the case of a contingent claim that depends on the first default among a list of $N$ credit events (such an option is called a first-to-default). For simplicity, we assume here that the default of each credit event is given by the same Weibull survival function. In Figure 5, we have represented the hazard rate, the survival function, the mean residual time-until-default and the density ${ }^{13}$.
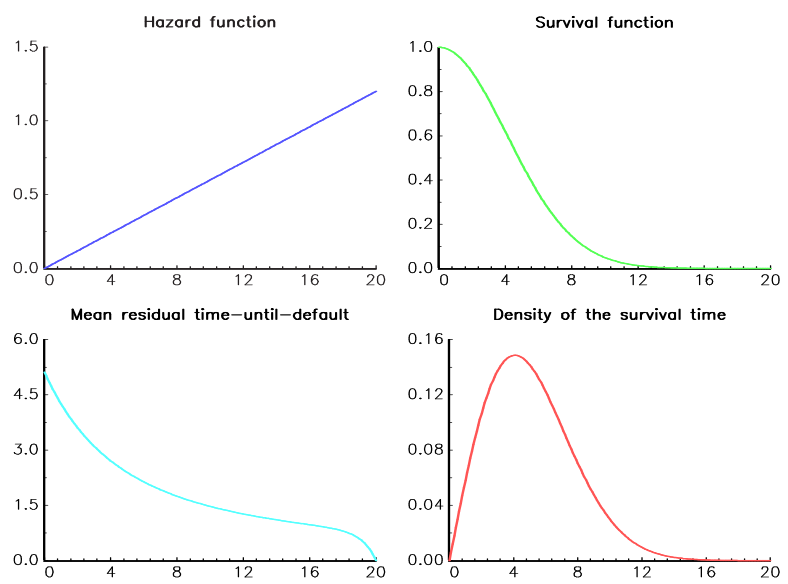

Figure 5: Weibull survival time.

Let us define the first-to-default time $\tau$ as follows

$$
\tau=\min \left(T_{1}, \ldots, T_{N}\right)
$$

NELSEN [1999] shows that the survival function of $\tau$ is given by the diagonal section of the survival copula ${ }^{14}$ :

$$
\mathbf{S}(\tau)=\breve{\mathbf{C}}\left(\mathbf{S}_{1}(\tau), \ldots, \mathbf{S}_{N}(\tau)\right)
$$

\footnotetext{
${ }^{12}$ For example, if the factors are Gamma distributed, the dependence function between defaults is the Cook-Johnson copula.

${ }^{13} \mathrm{We}$ assume that the baseline hazard is constant and equal to $3 \%$ per year and that the Weibull parameter is 2 .

${ }^{14}$ Note that density of $\tau$ is then given by

$$
f(\boldsymbol{\tau})=\sum_{n=1}^{N} \partial_{n} \breve{\mathbf{C}}\left(\mathbf{S}_{1}(\boldsymbol{\tau}), \ldots, \mathbf{S}_{N}(\boldsymbol{\tau})\right) \times f_{n}(\boldsymbol{\tau})
$$
}
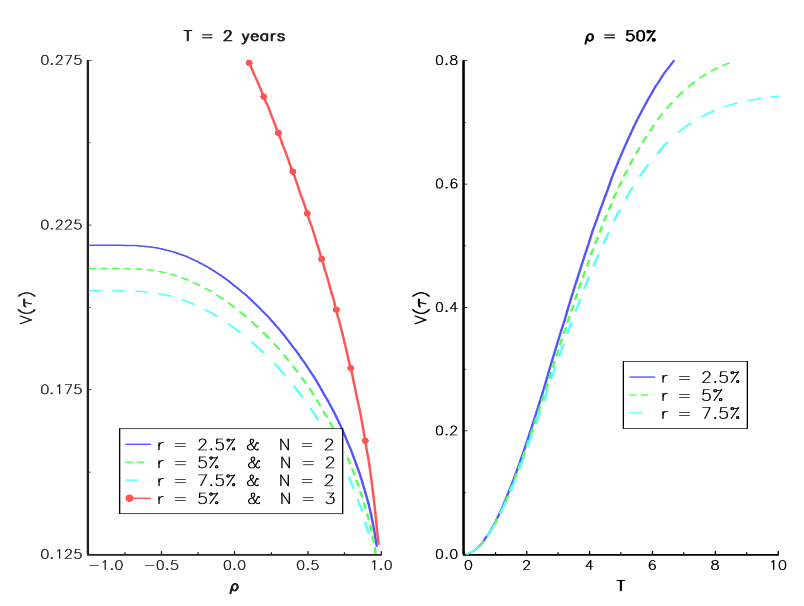

Figure 7: Premium of the first-to-default option.

Figure 6 shows the influence of the correlation parameter $\rho$ of the Normal copula and the influence of the number of securities $N$ on the density of $\tau$. In Figure 7 , we have reported the premium of the first-todefault option $\mathbf{1}_{[\tau \leq T]}$ in the case of deterministic interest rates. In the left plot, the maturity of the option $T$ is two years. In the right plot, we take two securities. As noted by Coutant, Martineu, Messines, Riboulet and RoncAlli [2001], we can find an analytical formula for the density of $\tau$ in the case of the Normal copula and compute easily the option prices even if the interest rates are stochastic thanks to numerical quadrature integration.
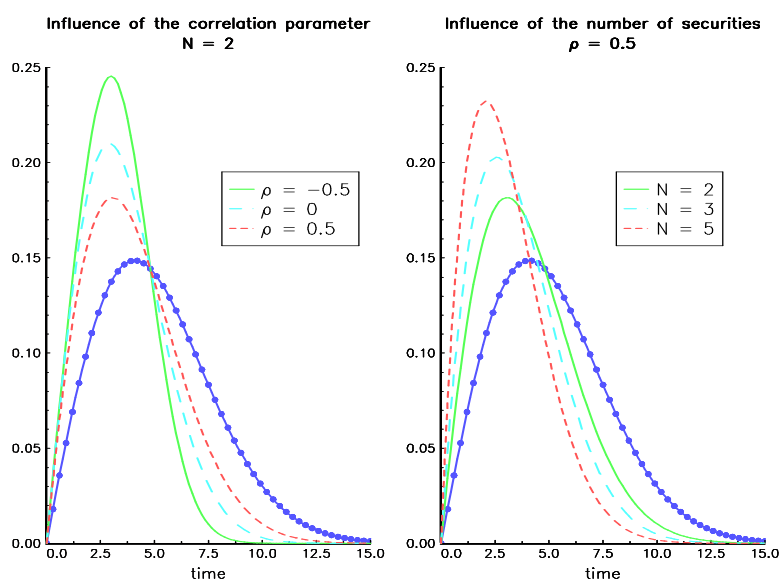

Figure 6: Density of the first-to-default. The solid line with circles corresponds to the density of one survival time.

where $f_{n}$ is the density of the survival time $T_{n}$. 


\section{Operational risk management}

One of the standard measurement methodology for operational risk with internal data is the following ${ }^{15}$ :

- Let $\zeta$ be the random variable that describes the severity of loss. We define also $\zeta^{k}(t)$ as the random process of $\zeta$ for each operational risk $k$ $(k=1, \ldots, K)$.

- For each risk, we assume that the number of events at time $t$ is a random variable $N_{k}(t)$.

- The loss process $\varrho(t)$ is also defined as

$$
\varrho(t)=\sum_{k=1}^{K} \sum_{j=1}^{N_{k}(t)} \zeta_{j}^{k}(t)
$$

- The Economic Capital with an $\alpha$ confidence level is usually defined as

$$
\mathrm{EC}=\mathbf{F}^{-1}(\alpha)
$$

with $\mathbf{F}^{-1}$ the inverse function of the loss distribution $\varrho(t)$. This methodology can be viewed as the Loss Distribution Approach proposed by the Basel Committee on Banking Supervision (see document [1]). In the New Basel Capital Accord, dependence effects in operational risk are not considered:

The capital charge is based on the simple sum of the operational risk VaR for each business line/risk type cell. Correlation effects across the cells are not considered in this approach (annex 6 of [1]).

But, from the point of view of economic capital allocation, "correlation effects" are a keypoint of the operational risk measure. One possibility is then to introduce dependence by using correlations between frequencies of different types of risk. Each individual frequency $N_{k}(t)$ is generally assumed to be a Poisson variable $\mathcal{P}$ with mean $\lambda_{k}$. However, multivariate Poisson distributions are relatively complicated for dimensions higher than two. Song [2000] suggests then an interesting alternative by using copulas. Assuming a Normal copula, we note $\mathcal{P}(\boldsymbol{\lambda}, \boldsymbol{\rho})$ the multivariate Poisson distribution generated by the Normal copula with parameter $\boldsymbol{\rho}$ and univariate Poisson distribution $\mathcal{P}\left(\lambda_{k}\right)$. The next table contains the probability mass function $p_{i, j}=\operatorname{Pr}\left\{N_{1}=i, N_{2}=j\right\}$ of the bivariate Poisson distribution $P\left(\lambda_{1}=1, \lambda_{2}=1, \rho=0.5\right)$.

\footnotetext{
${ }^{15}$ We assume that time is discrete $-t \in \mathbb{N}$ - and that the period of reference is one.
}

\begin{tabular}{c|cccc|c}
$p_{i, j}$ & 0 & 1 & 2 & $\cdots$ & $p_{i,}$ \\
\hline 0 & 0.095 & 0.133 & 0.089 & & 0.368 \\
1 & 0.034 & 0.100 & 0.113 & & 0.368 \\
2 & 0.006 & 0.031 & 0.052 & & 0.184 \\
$\vdots$ & & & & & $\vdots$ \\
\hline$p \cdot, j$ & 0.135 & 0.271 & 0.271 & $\cdots$ & 1
\end{tabular}

If $\rho=-0.5$, we obtain the following values for $p_{i, j}$.

\begin{tabular}{c|cccc|c}
$p_{i, j}$ & 0 & 1 & 2 & $\cdots$ & $p_{i,}$ \\
\hline 0 & 0.014 & 0.062 & 0.101 & & 0.368 \\
1 & 0.044 & 0.112 & 0.111 & & 0.368 \\
2 & 0.044 & 0.068 & 0.046 & & 0.184 \\
$\vdots$ & & & & & $\vdots$ \\
\hline$p \cdot, j$ & 0.135 & 0.271 & 0.271 & $\cdots$ & 1
\end{tabular}

The Economic Capital $\mathrm{EC}=\mathbf{F}^{-1}(\alpha)$ with an $\alpha$ confidence level for operational risk could then be calculated by assuming that $\mathbf{N}=\left\{N_{1}, \ldots, N_{K}\right\}$ follows a multivariate Poisson distribution $\mathcal{P}(\boldsymbol{\lambda}, \boldsymbol{\rho})$. Moreover, there are no computational difficulties, because the estimation of the parameters $\boldsymbol{\lambda}$ and $\boldsymbol{\rho}$ is straightforward and the quantile can be easily obtained with Monte Carlo methods. Figure 8 illustrates the simulation of a bivariate Poisson distribution.
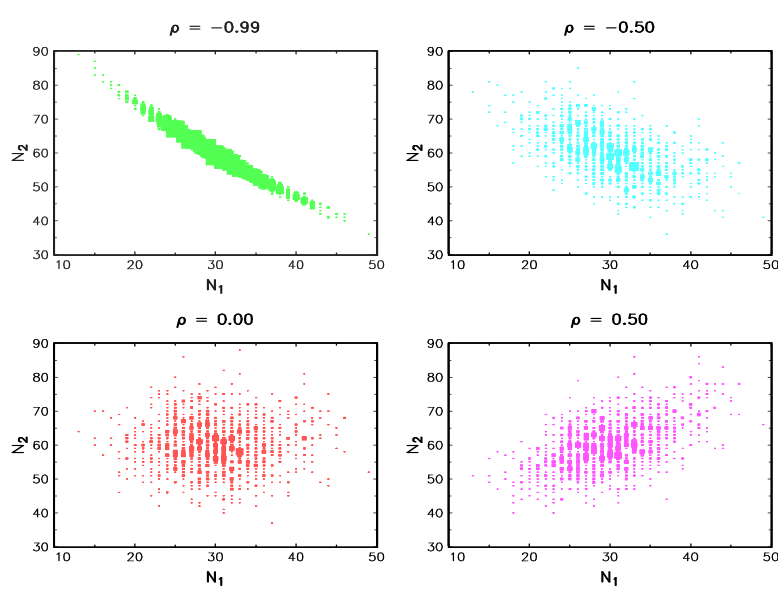

Figure 8: Random generation of bivariate Poisson variates $\mathcal{P}(30)$ and $\mathcal{P}(60)$.

\section{Conclusion}

In this paper, we show that copula is a very powerful tool for risk management since it fulfills one of its main goal: the modelling of dependence between the individual risks. That is why this approach is an open field for 
risk. Indeed, there is a need to find other 'industrial' copula functions such as Normal and Student. Before going further, copulas have to become more familiar to practitioners and we believe they will.

Eric Bouyé, FERC, City University Business School, England

Valdo Durrleman, Department of Operations Research \& Financial Engineering, Princeton University, USA

Ashkan Nikeghbali, Université Paris VI, France

Gaël Riboulet \& Thierry Roncalli, Groupe de Recherche Opérationnelle, Crédit Lyonnais, France

\section{References}

[1] Basel Committee on Banking Supervision, Operational Risk - Consultative Document, Supporting document to the New Basel Capital Accord, January 2001

[2] Bouyé, E., V. Durrleman, A. Nikeghbali, G. Riboulet and T. Roncalli [2000], Copulas for finance - a reading guide and some applications, Groupe de Recherche Opérationnelle, Crédit Lyonnais, Working Paper

[3] Coutant, S., P. Martineu, J. Messines, G. Riboulet and T. Roncalli [2001], Revisiting the dependence in credit risk models, Groupe de Recherche Opérationnelle, Crédit Lyonnais, Working Paper

[4] Deheuvels, P. [1978], Caractérisation complète des lois extrêmes multivariées et de la convergence des types extrêmes, Publications de l'Institut de Statistique de l'Université de Paris, 23, 1-36

[5] Durrleman, V., A. Nikeghbali and T. RonCALLI [2000], Which copula is the right one?, Groupe de Recherche Opérationnelle, Crédit Lyonnais, Working Paper

[6] Embrechts, P., McNeil, A.J. and D. StrauMANN [1999], Correlation: pitfalls and alternatives, Risk magazine, 12, May, 69-71

[7] Embrechts, P., McNeil, A.J. and D. StrauMANN [2000], Correlation and dependency in risk management: properties and pitfalls, in M. Dempster and H.K. Moffatt (Eds.), Risk Management:
Value at Risk and Beyond, Cambridge University Press (yet to appear)

[8] Genest, C., K. Ghoudi and L-P Rivest [1995], A semiparametric estimation procedure for dependence parameters in multivariate families of distributions, Biometrika, 82, 543-552

[9] Georges, P., A-G. Lamy, E. Nicolas, G. Quibel and T. Roncalli [2001], Multivariate survival modelling: a unified approach with copulas, Groupe de Recherche Opérationnelle, Crédit Lyonnais, Working Paper, in preparation

[10] Joe, H. [1997], Multivariate Models and Dependence Concepts, Monographs on Statistics and Applied Probability, 73, Chapmann \& Hall, London

[11] LI, D.X. [2000], On default correlation: a copula function approach, Journal of Fixed Income, March, 43-54

[12] Maccarinelli, M. and V. Maggiolini [2000], The envolving practice of credit risk management in global financial institutions, Risk Conference, 26/27 September, Paris

[13] Nelsen, R.B. [1999], An Introduction to Copulas, Lectures Notes in Statistics, 139, Springer Verlag, New York

[14] Song, P. X-K. [2000], Multivariate dispersion models generated from Gaussian copula, Scandinavian Journal of Statistics, 27-2, 305-320 